NTP RESEARCH REPORT ON

SYNTHETIC TURF/RECYCLED TIRE CRUMB RuBber: 14-DAY

EXPOSURE ChARACTERIZATION

StUdies OF CRUMB RUBBER IN

Female Mice Housed on Mixed

BEDDING OR DOSED VIA FEED OR

Oral GaVAGE

NTP RR 14

JULY 2019 


\title{
NTP Research Report on Synthetic Turf/Recycled Tire Crumb Rubber: 14-Day Exposure Characterization Studies of Crumb Rubber in Female Mice Housed on Mixed Bedding or Dosed via Feed or Oral Gavage
}

Research Report 14

National Toxicology Program

July 2019

\author{
National Toxicology Program \\ Public Health Service \\ U.S Department of Health and Human Services \\ ISSN: 2473-4756 \\ Research Triangle Park, North Carolina, USA
}




\section{Table of Contents}

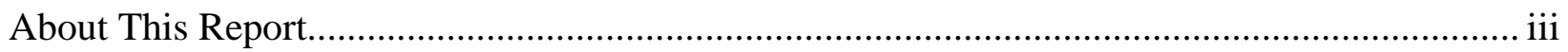

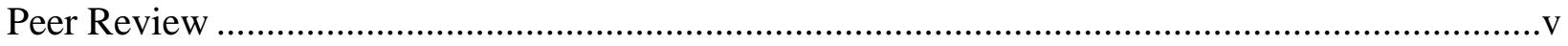

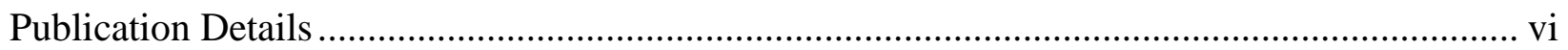

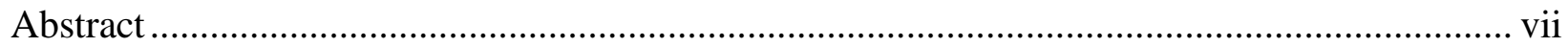

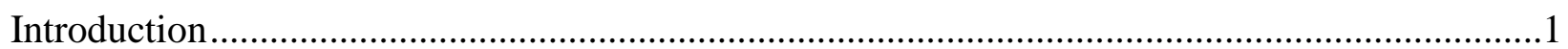

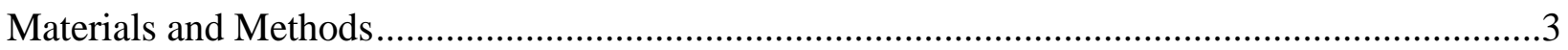

Crumb Rubber Handling and Characterization ....................................................................

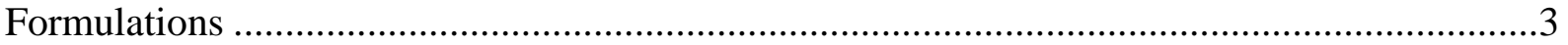

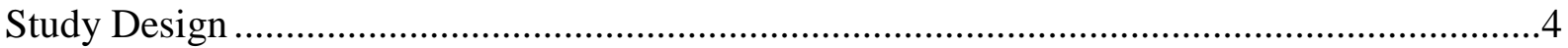

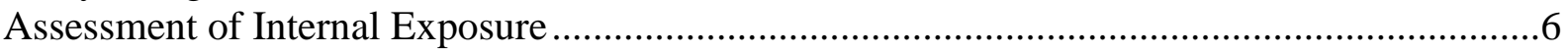

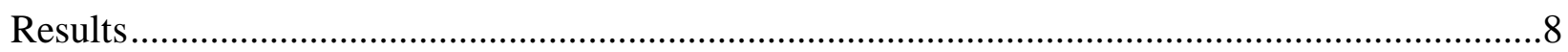

Survival, Body Weights, and Clinical Observations .............................................................

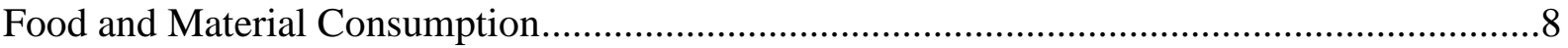

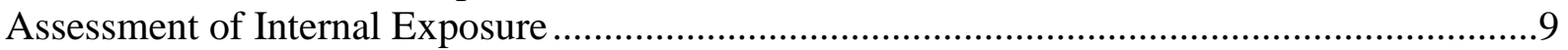

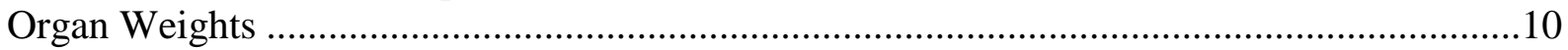

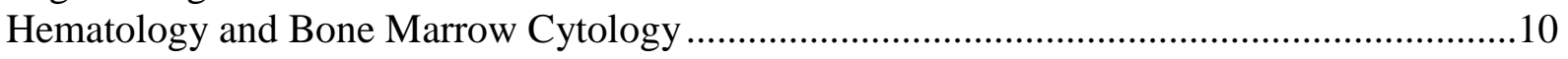

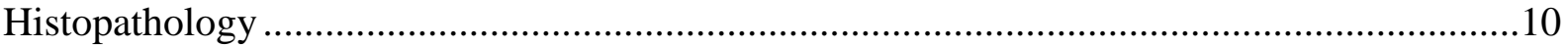

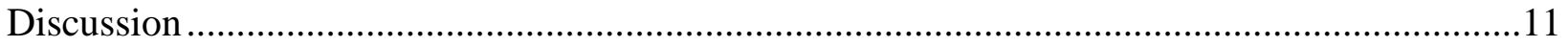

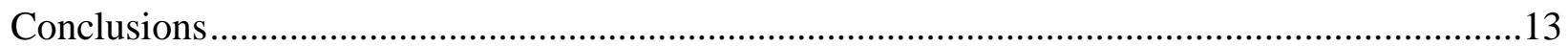

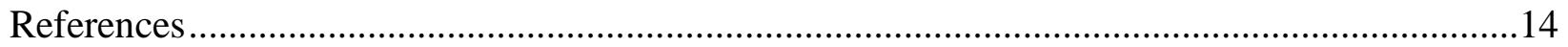

\section{Tables}

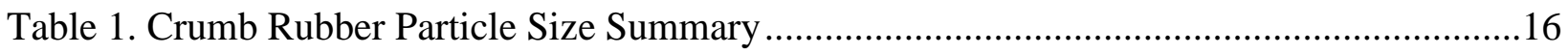

Table 2. Consumption of Crumb Rubber in 50,000 ppm Dosed Feed Group ...............................17

Table 3. Untargeted Analysis..............................................................................................18

\section{Figures}

Figure 1. Image of Crumb Rubber Particles: Lot CRM09132016, 400 Mesh...............................19

Figure 2. Image of Crumb Rubber Particles: Lot CRM 12052016, Combined ..............................19

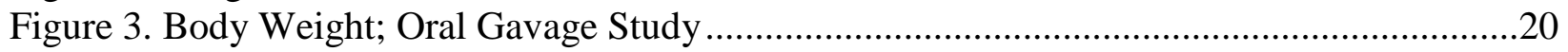

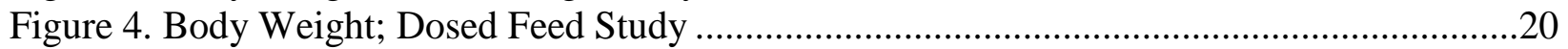

Figure 5. Body Weight; Mixed Bedding Study ......................................................................20

Figure 6. Principal Component Analysis of Plasma and Urine Untargeted Data; Oral

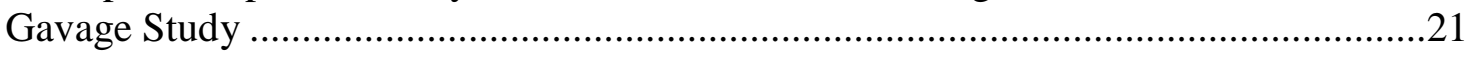

Figure 7. Principal Component Analysis of Plasma and Urine Untargeted Data; Dosed

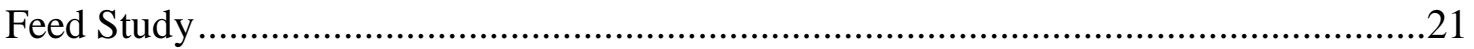

Figure 8. Principal Component Analysis of Plasma and Urine Untargeted Data; Mixed

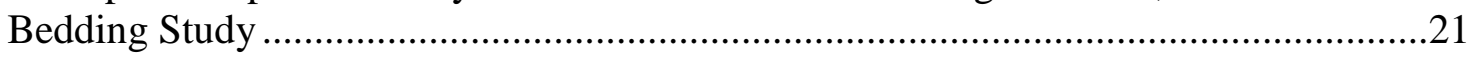




\section{About This Report}

National Toxicology Program ${ }^{1}$

${ }^{1}$ Division of the National Toxicology Program, National Institute of Environmental Health Sciences, Research Triangle Park, North Carolina, USA

\section{Collaborators}

Georgia K. Roberts, Karen E. Elsass, Suramya Waidyanatha, Tim Fennell, Michelle C. Cora, Amy E. Brix, Michelle J. Hooth, Scott A. Masten, Nigel J. Walker, William M. Gwinn, Barney R. Sparrow, Jamie S. Richey, Dawn M. Fallacara, Heather M. Toy, Matthew D. Stout

Division of the National Toxicology Program, National Institute of Environmental Health Sciences, Research Triangle Park, North Carolina, USA

Designed study, evaluated and interpreted study findings

Georgia K. Roberts, Ph.D.

Michelle C. Cora, D.V.M.

William M. Gwinn, Ph.D.

Michelle J. Hooth, Ph.D.

Scott A. Masten, Ph.D.

Matthew D. Stout, Ph.D.

Suramya Waidyanatha, Ph.D.

Nigel J. Walker, Ph.D.

Battelle Memorial Institute, Columbus, Ohio, USA

Conducted 14-day studies and evaluated pathology findings

Barney R. Sparrow, Ph.D., Principal Investigator

Karen E. Elsass, B.S., Study Director

Dawn M. Fallacara, Ph.D.

Jamie S. Richey, M.S.

Heather M. Toy, B.S.

RTI International, Durham, North Carolina, USA

Analyzed biological samples

Tim Fennell, Ph.D.

EPL, Inc., Research Triangle Park, North Carolina, USA

Provided pathology review

Amy E. Brix, Ph.D., D.V.M. 


\section{Contributors}

Division of the National Toxicology Program, National Institute of Environmental Health Sciences, Research Triangle Park, North Carolina, USA

Provided overall programmatic guidance and review

Abee L. Boyles, Ph.D.

John R. Bucher, Ph.D.

Michael J. DeVito, Ph.D.

Darlene Dixon, Ph.D.

Dave E. Malarkey, Ph.D., D.V.M.

Cynthia V. Rider, Ph.D.

Provided internal peer review

Anika L. Dzierlenga, Ph.D.

Suzanne E. Fenton, Ph.D.

Esra Mutlu, Ph.D.

Provided oversight of the external peer review

Mary S. Wolfe, Ph.D.

\section{ICF, Durham, North Carolina, USA}

Prepared reports and conducted peer review

David F. Burch, M.E.M., Principal Investigator

Susan E. Blaine, B.A.

Natalie K. Blanton, M.P.H.

Lindsey M. Green, M.P.H.

Katherine R. Helmick, M.P.H.

Tara Hamilton, M.S.

Penelope E. Kellar, M.S.

Whitney K. Mitchell, B.S 


\section{Peer Review}

The draft research report, NTP Research Report on Synthetic Turf/Recycled Tire Crumb Rubber: 14-Day Exposure Characterization Studies of Crumb Rubber in Female Mice Housed on Mixed Bedding or Dosed via Feed or Oral Gavage, was evaluated by the reviewers listed below. These reviewers served as independent scientists, not as representatives of any institution, company, or governmental agency. In this capacity, reviewers determined if the design and conditions of these NTP studies were appropriate and ensured that this NTP Research Report presented the experimental results and conclusions fully and clearly.

\section{Peer Reviewers}

\section{William J. Brock, Ph.D., DABT, Fellow ATS}

Owner

Brock Scientific Consulting, LLC Montgomery Village, Maryland, USA

\section{Joshua Kellogg, Ph.D.}

Postdoctoral Research Fellow

University of North Carolina at Greensboro

Greensboro, North Carolina, USA

Kristini K. Miles, Ph.D., DABT

President

Venture Chemical Consulting, LLC

Atlanta, Georgia, USA 


\section{Publication Details}

Publisher: National Toxicology Program

Publishing Location: Research Triangle Park, North Carolina

ISSN: 2473-4756

DOI: https://doi.org/10.22427/NTP-RR-14

Report Series: NTP Research Report Series

Report Series Number: 14

Official citation: National Toxicology Program (NTP). 2019. NTP research report on synthetic turf/recycled tire crumb rubber: 14-day exposure characterization studies of crumb rubber in female mice housed on mixed bedding or dosed via feed or oral gavage. Research Triangle Park, NC: National Toxicology Program. Research Report 14. 


\section{Abstract}

Public health concern for playing on synthetic turf fields with crumb rubber infill has increased in recent years. Crumb rubber manufactured from recycled automobile tires contains potential carcinogenic and toxic substances, and, with over 12,000 synthetic turf fields in the United States, the potential for exposure is widespread. The National Toxicology Program (NTP) conducted research to improve the understanding of potential human exposure to crumb rubber and its biological activity. The objective of the in vivo studies as described in this report was to determine if there was evidence of systemic exposure to crumb rubber constituents following exposure to crumb rubber in experimental animals.

Crumb rubber was obtained from multiple commercial sources, combined into a single lot, and size fractionated for use in these studies: $37-170 \mu \mathrm{m}$ for oral gavage and $\geq 170 \mu \mathrm{m}$ for feed and bedding studies. NTP conducted 14-day studies in female $\mathrm{B} 6 \mathrm{C} 3 \mathrm{~F} 1 / \mathrm{N}$ mice by oral gavage (0 or $1,250 \mathrm{mg} / \mathrm{kg} /$ day in corn oil), dosed feed (0 or 50,000 ppm), or by housing on crumb rubber mixed bedding (bedding only or 50:50 wt:wt). Plasma and urine were collected from all groups to determine internal exposure. Hematology, bone marrow cytology, and limited histopathology were evaluated as conventional approaches to assess systemic exposure through evidence of biological effect.

Untargeted chemical analysis of urine and plasma revealed no apparent difference in chemical profiles between control and treated animals using principal component analysis, for any route of exposure. While overall chemical profiles were not affected, some constituents potentially originating from crumb rubber were detected at higher levels in urine or plasma of crumb rubber-treated animals, compared to controls. No biological relevant effects were observed on survival, food consumption, body weight, or organ weights following crumb rubber exposure by any route tested. Small changes in hematology parameters were observed in crumb rubbertreated groups, but none of these changes was considered biologically meaningful. No histopathologic lesions were observed in animals exposed to crumb rubber via dosed feed or mixed bedding. Animals dosed with crumb rubber by oral gavage had higher occurrences of esophageal inflammation compared to the respective controls; the average severity of this lesion was similar between controls and the crumb rubber gavage group. In conjunction with chemical characterization and in vitro testing of the same material, this work will contribute to what is known about potential human exposure to crumb rubber constituents and biological effects resulting from contact with crumb rubber used in synthetic turf. 


\section{Introduction}

Synthetic turf fields are widely used in the United States, and their use is expanding. Although such fields historically have been installed in professional sports complexes, they are becoming increasingly popular in community recreational areas, including schools and public parks. These expanded applications include areas across the United States that are used by people of all ages, including young children. In a synthetic turf field, infill materials are spread between the "grass" fibers to provide cushioning and traction. Today, most commonly, the infill consists of granulated rubber pellets referred to as crumb rubber. Crumb rubber is manufactured by shredding used or recycled automobile tires. Recycled tires contain numerous, potentially carcinogenic and toxic substances, either as components of the original tire rubber or accumulated during normal use. While information is known about tire manufacturing, many variables are unknown, including persistence of byproducts, chemical changes during vulcanization, and adsorption of environmental chemicals, which might affect the chemical composition of the ultimate crumb rubber product. A recent literature review of crumb rubber chemical characterization studies outlines some chemicals of concern; using ADMET Predictor $^{\mathrm{TM}}$, this study identified 197 predicted carcinogens, of which 52 have been previously classified as carcinogens by the U.S. Environmental Protection Agency (EPA) or European Chemicals Agency (ECHA) ${ }^{1}$. Other chemicals of concern as well as more information about crumb rubber manufacturing and synthetic turf construction and standards, are described in the 2016 EPA Federal Research Action Plan².

Public concerns about potential health impacts associated with the use of synthetic turf fields have risen dramatically in recent years due to the chemical composition of crumb rubber and the opportunity for widespread, frequent, and long-term exposure, particularly for young athletes. Considering that information to evaluate whether there are adverse health effects from playing on synthetic turf fields is currently limited, several governmental organizations at the international, federal, and state level recently launched research efforts to better understand human exposure and human health hazards focusing on crumb rubber. Internationally, $\mathrm{ECHA}^{3}$ and the National Institute for Public Health and the Environment (RIVM) ${ }^{4}$ of the Netherlands have evaluated the health risks of playing sports on synthetic turf with crumb rubber. The Federal Research Action Plan ${ }^{5-7}$, which involves several U.S. federal agencies, aims to determine and fill important knowledge gaps (FRAP 2016, Appendix B), characterize constituents of recycled tire crumb, and identify ways that people are exposed to tire crumb rubber through typical field use activities. The California Office of Environmental Health Hazard Assessment $(\mathrm{OEHHA})^{8}$ is evaluating exposure scenarios, characterizing new and in-field crumb rubber, and developing biomonitoring protocols.

In 2015, OEHHA nominated synthetic turf/crumb rubber to the National Toxicology Program (NTP) for short-term in vivo and in vitro studies to generate information and improve understanding of potential health impacts of chemicals released from synthetic turf, with an emphasis on crumb rubber. The NTP research program ${ }^{9}$ developed in response to this request used in vitro and in vivo systems to address uncertainties about potential human exposure to hazardous components of crumb rubber. The NTP research approach included both in vivo and in vitro studies and considered the most likely routes of human contact with crumb rubber (e.g., ingestion, dermal contact, and inhalation). The study objective was to investigate which exposure 
conditions could have biological effects, with a focus on characterizing the bioaccessibility and systemic exposure of crumb rubber constituents.

The results of the NTP studies on crumb rubber are communicated in a series of Research Reports ${ }^{10-13}$. The crumb rubber used in the NTP studies was provided by OEHHA specifically for research purposes, and consisted of fresh recycled tire crumb rubber obtained from manufacturing facilities. The physical and chemical characteristics of the material were evaluated using a variety of analytical methods ${ }^{10}$. The characterization work provided an understanding of the material used in the NTP studies and helped interpret chemical analyses in the in vivo and in vitro studies. Characterization of the NTP material will allow interpretation of NTP study findings in relation to crumb rubber evaluated as part of the Federal Research Action Plan (FRAP) and OEHHA research efforts, including samples from new and weathered material, and samples from indoor and outdoor fields. For in vitro testing, multiple cell lines (representing skin, lung, and small intestine) were used to evaluate the extractability of crumb rubber and to look for evidence of biological activity of crumb rubber constituents via measurements of cytotoxicity ${ }^{11}$. For in vivo studies, feasibility testing was conducted to determine what routes of exposure were possible ${ }^{12}$. On the basis of this testing, 14-day in vivo studies were performed ${ }^{13}$ using oral exposure and exposure in bedding. Dermal studies were not conducted on the basis of feasibility work ${ }^{12}$, and feasibility testing for particle and vapor inhalation studies is ongoing.

The objective of the in vivo studies was to determine if there was evidence of systemic exposure to crumb rubber constituents following exposure to crumb rubber. Based on the scope and objective of these studies, a single sex/species and a single dose level per route was selected for evaluation. Female B6C3F1/N mice were administered size fractionated crumb rubber for 14 days by oral gavage ( 0 or $1,250 \mathrm{mg} / \mathrm{kg}$, corn oil vehicle; 37-170 $\mu \mathrm{m})$, dosed feed ( 0 or $50,000 \mathrm{ppm} ;>170 \mu \mathrm{m}$ ), or mixed bedding (bedding only or 50:50 wt:wt, bedding:crumb rubber; $>170 \mu \mathrm{m}$ ). Chemical analysis of biological fluids (urine and plasma) and conventional toxicological endpoints (hematology, bone marrow cytology, histopathology) were used to determine if there were biological effects as evidence of systemic exposure. 


\section{Materials and Methods}

\section{Crumb Rubber Handling and Characterization}

OEHHA provided the crumb rubber used in these studies. The material, manufactured by either an ambient or a cryogenic manufacturing process, is fresh crumb rubber. Three lots were received in multiple 1-L glass jars. First, the material from one facility and one type of processing was combined to produce three lots, each approximately $5 \mathrm{~kg}$. Subsequently, all material was combined into one lot (Lot No. CRM06092016). Physical and chemical characterization of this lot is described elsewhere ${ }^{10}$.

Previous studies were performed to determine the feasibility of exposing animals to crumb rubber by various routes of exposure. The material was size fractionated (mesh sizes 400, 80, 40, and 14) to optimize exposure via various routes ${ }^{10}$. Following completion of the feasibility studies, the three larger particle size fractions (mesh sizes 80,40, and 14) were combined, resulting in two fraction lots [Lot No. CRM12052016 (combined mesh; >170 $\mu \mathrm{m}$ ) and Lot No. CRM09132016 (400 mesh; 37-170 $\mu \mathrm{m}$ )]; both lots were stored refrigerated. Chemical profiling of the two lots was conducted in comparison to the bulk material ${ }^{10}$.

For the current study, particle size was evaluated on both lots (Table 1). Size evaluation of Lot No. CRM12052016 (combined mesh) was determined by optical microscopy using an Olympus (Tokyo, Japan) SZK12 stereomicroscope. Particle size evaluation of Lot No. CRM09132016 (400 mesh) was determined by scanning electron microscopy (SEM) using a JEOL (Peabody, MA) JSM-7600F. These results are consistent with the estimated particle size ranges following sieving. Representative digital images of each lot are shown in Figure 1 and Figure 2.

Chemical analysis (metals, volatiles) of the combined and 400 mesh fractions was conducted and compared to the bulk material, and is summarized in Research Report 11 on the Chemical and Physical Characterization ${ }^{10}$ of the materials used in the NTP studies.

\section{Formulations}

Formulations of crumb rubber for the oral gavage study (Lot No. CRM09132016, 400 mesh) were prepared in corn oil at concentrations of 0 (corn oil only) and $125 \mathrm{mg} / \mathrm{mL}$. Corn oil was purchased from Spectrum Chemical Manufacturing Corporation (New Brunswick, NJ; Lot No. 2FA0334). Corn oil was analyzed for peroxide content and levels were below $1.5 \mathrm{meq} / \mathrm{kg}$. Gavage formulations were prepared weekly, stored under refrigeration $\left(2\right.$ to $\left.8^{\circ} \mathrm{C}\right)$, and used within 10 days after formulation. Prior to dosing, gavage formulations were allowed to come to ambient temperature and mixed for 15 minutes using a stir bar and stir plate; formulations were constantly stirred during the time of dose administration.

Formulations of crumb rubber for the dosed feed and mixed bedding studies (Lot No. CRM12052016, combined material) were prepared on a per-cage basis. For the dosed feed study, formulations were prepared at concentrations of 0 (feed only) or 50,000 ppm in irradiated NTP-2000 meal feed (Zeigler, Gardners, PA). Formulations for the mixed bedding study were prepared at concentrations of 0:100 or 50:50 (wt:wt) crumb rubber/bedding in irradiated hardwood bedding (Sani-Chips, P.J. Murphy Forest Products Corporation, Montville, NJ). 
Synthetic Turf/Recycled Tire Crumb Rubber:

Characterization of the Biological Activity of Crumb Rubber In Vitro

Formulations for dosed feed and mixed bedding were prepared fresh on Days 0, 4, 7, and 11 by manually mixing until visually homogenous and then immediately presented to the animals.

\section{Study Design}

Several aspects of the study design, including sex/species and exposure concentrations, were selected based on logistical considerations relating to test article availability. Mice, rather than rats, were selected for the gavage studies to conserve test material. For the dosed feed and mixed bedding studies, female mice (can be group housed) were selected, instead of male mice (require individual housing) or rats (group housed except with larger cage area and feed requirements). For consistency across routes of exposure, female mice were selected for all studies. The dose for the gavage studies was selected based on availability of 400-mesh crumb rubber (Lot No. CRM09132016). For dosed feed, 50,000 ppm was selected as the exposure concentration as a limit dose; 50,000 ppm or 5\% of the diet is considered the highest concentration, or limit dose, that should be used to retain adequate nutritional value of the feed. For the bedding studies, a 50:50 (wt:wt) bedding mixture was selected to provide a high environmental exposure, while still including absorbent bedding; this mixture extended the length of time between cage changes, from daily to twice weekly, conserving crumb rubber material.

The studies presented here were conducted at Battelle Memorial Institute (West Jefferson, OH). Animal care and use was in accordance with the U.S. Public Health Service policy on humane care and use of laboratory animals and the Guide for the Care and Use of Laboratory Animals ${ }^{14}$. All animal studies were conducted in an animal facility accredited by the Association for the Assessment and Accreditation of Laboratory Animal Care International. Studies were approved by the Battelle Animal Care and Use Committee and conducted in accordance with all relevant National Institutes of Health (NIH) and NTP animal care and use policies, the Specifications for the Conduct of Studies to Evaluate the Toxic and Carcinogenic Potential of Chemical, Biological and Physical Agents in Laboratory Animals for the National Toxicology Program ${ }^{15}$, and applicable federal, state, and local regulations and guidelines.

Female B6C3F1/N mice were supplied from the NTP colony at Taconic Biosciences (Germantown, NY). Upon arrival, animals were quarantined for 8 days and provided ad libitum access to irradiated NTP-2000 wafer feed (Zeigler Brothers, Inc., Gardeners, PA) and tap water. Following quarantine, 8- to 9-week-old animals were randomized into one of six groups by body weight. Animals were assigned to the control or exposed group for each route of exposure: oral gavage, dosed feed, or mixed bedding. Animals were uniquely identified by tail tattoo upon randomization and housed five per cage. Housing conditions were maintained at temperatures of $72^{\circ} \pm 3^{\circ} \mathrm{F}$ and relative humidity of $50 \% \pm 15 \%$. Fluorescent lighting was controlled on an automatic light cycle of 12 hours on/off per day.

Groups of 15 female B6C3F1/N mice were exposed to crumb rubber by oral gavage ( 0 or $1250 \mathrm{mg} / \mathrm{kg}$ ), dosed feed (0 or 50,000 ppm), or mixed bedding (0:100 or 50:50 wt:wt crumb rubber/bedding) for 14 consecutive days. Ten animals in each group were assigned to the core study and five were assigned to a biological sampling cohort; these group sizes were selected based on historical experience in detecting effect and interpreting data for the respective endpoints. Beginning on Day 0, animals in the oral gavage study were dosed with corn oil (vehicle, $0 \mathrm{mg} / \mathrm{kg}$ ) or crumb rubber $(1,250 \mathrm{mg} / \mathrm{kg} /$ day in corn oil); doses were calculated based on the animal's most recent weight. Animals in the dosed feed study were given ad libitum 
access to NTP-2000 meal feed, containing 0 ppm crumb rubber, or 50,000 ppm crumb rubber; animals in the oral gavage and mixed bedding studies remained on NTP-2000 wafer feed. Animals in the mixed bedding study were placed on 0:100 or 50:50 wt:wt. crumb rubber/bedding. Animals were observed for moribundity/mortality and clinical observations twice daily. Body weights and food consumption were recorded at study start (Day 0), twice weekly, and at study termination.

On Day 6, animals in the biological sampling cohort were transferred to metabolism cages and individually housed for overnight urine collection. Feces were not saved or analyzed due to the likely presence of undigested crumb rubber particles. While in metabolism cages, animals in the dosed feed study (50,000-ppm group) were provided control meal feed to prevent contamination of collected samples. Following overnight urine collection, animals were returned to their home cages and continued exposure. On Day 13, immediately following lights on for bedding and dosed feed groups and 2 hours post-gavage for gavage groups, animals in the biological sampling cohort were anesthetized with $70 \% / 30 \% \mathrm{CO}_{2} / \mathrm{O}_{2}$, and blood was collected via cardiac puncture into tubes containing $\mathrm{K}_{3}$ EDTA. Blood was processed to collect plasma. Plasma and urine were analyzed as described below to assess internal exposure

On Day 14, blood was collected via the retro-orbital sinus from core study animals anesthetized with $70 / 30 \% \mathrm{CO}_{2} / \mathrm{O}_{2}$ into tubes containing $\mathrm{K}_{3}$ EDTA. The following analyses were performed using an Advia 120 hematology analyzer (Siemens Medical; City, State): erythrocyte, reticulocyte, and platelet counts; total white blood cell count and differential; hematocrit; hemoglobin concentration; mean cell volume; and mean cell hemoglobin concentration. A manual hematocrit was performed with the use of a microcentrifuge and capillary reader. Blood smears were prepared and stained with a Wright-Giemsa stain and the morphology of erythrocytes, white blood cells and platelets evaluated; nRBCs were enumerated when present. Following blood collection, a gross necropsy then was performed on all core animals. Organ weights were recorded for liver, thymus, kidney, ovary, heart, and lungs. Tissues were collected for histopathology (adrenal glands, brain, clitoral glands, esophagus, eyes, femur-left, gallbladder, Harderian glands, heart and aorta, large and small intestine, kidneys, liver, lungs, lymph nodes, mammary gland, muscle, nerve, nose, oral cavity, pancreas, parathyroid glands, pituitary glands, salivary glands, spinal cord, spleen, stomach, thymus, thyroid gland, tongue, trachea, urinary bladder, uterus/cervix/vagina/ovaries, and Zymbal's gland). All collected tissues were preserved in $10 \%$ neutral-buffered formalin, trimmed and processed, embedded in paraffin, sectioned at $5 \mu \mathrm{m}$ and stained with hematoxylin and eosin. Bone marrow was flushed from the right femur using Hank's Balanced Salt Solution that was supplemented with $0.15 \% \mathrm{~K}_{3}$ EDTA and $5 \%$ bovine serum albumin. A total nucleated cell count of the bone marrow single cell suspension was determined using an Advia 120 hematology analyzer. From the suspension, cytospin slide preparations were made for the cytological evaluation of the bone marrow. Included in this evaluation was the morphologic assessment of all the bone marrow cell lines, a differential cell count of 500 cells, and calculation of a myeloid to erythroid (M:E) ratio. Bone marrow cytology is not a standard endpoint evaluated in NTP studies, but was included here due to relevance to endpoints of concern in humans.

In-life data (body weights, food consumption, clinical observations), histopathology, hematology, and bone marrow cytology data were analyzed by first using the Levene test for homogeneity of variances and the Shapiro-Wilk test for normality of distributions. Assuming normal distribution, Dunnett's pairwise test was used to determine a statistical difference 
between groups. If the Levene test or Shapiro-Wilk test failed, a Wilcoxon/Bonferroni-Holm test was run.

\section{Assessment of Internal Exposure}

Plasma samples $(n=4-5)$ and urine samples $(n=3-5)$ were each classified into six groups according to the exposure route and treatment group: oral gavage ( 0 or $1,250 \mathrm{mg} / \mathrm{kg})$, dosed feed ( 0 or $50,000 \mathrm{ppm}$ ) or mixed bedding (bedding only or 50:50 wt:wt). All samples were vortexed 2 minutes and centrifuged for 2 minutes. Pooled samples were made for each of the six groups for plasma and urine by mixing equal volumes of individual animal samples from a given group and processed as described above. A total pool representing all groups, for each plasma and urine sample, was made by mixing volumes of each of the six respective pools. The total pool served as a measure of overall variability. Buffer was added to precipitate protein, and supernatant was dried down and reconstituted in 95:5\% volume water:methanol.

Samples were vortexed and supernatant was analyzed using Waters Acquity Ultra Performance Liquid Chromatography (UPLC) and a Synapt G2-Si ESI-Q-TOF mass spectrometer. Chromatographic separation was accomplished on an Acquity HSST3 C18 column $(2.1 \times 100 \mathrm{~mm}, 1.8 \mu \mathrm{m})$ at $50^{\circ} \mathrm{C}$ using a gradient elution involving mobile phase A: $0.1 \%$ formic acid-water (v/v), and mobile phase B: $0.1 \%$ formic acid-methanol (v/v), using a flow rate of $400 \mu \mathrm{L} / \mathrm{min}$. The injection volume was $5 \mu \mathrm{L}$. The gradient consisted of $99 \% \mathrm{~A}$ and $1 \% \mathrm{~B}$ for 1 minute, a linear gradient from $1 \%$ B to $99 \%$ at 16 minutes, hold at $99 \%$ B until 20 minutes, with a linear gradient to return to $99 \% \mathrm{~A}$ at 20.5 minutes.

Spectra of samples were imported into Progenesis QI software (Nonlinear Dynamics, UK) according to sample matrix, exposure route, and mode (positive or negative). Progenesis QI (Nonlinear Dynamics, UK) aligned samples to an alignment reference, and picked unique peaks observed for the appropriate project.

Differentiating compounds ( $\mathrm{p}<0.1$, fold change $>2$ ) were determined from a data reduction method listed below, and the compounds flagged within Progenesis QI (Nonlinear Dynamics, UK). Identification of compounds was attempted using two approaches, untargeted and suspect screening. For the untargeted approach, compounds were searched against In-house Retention Time library, In-house Exogenous database, as well as the HMDB, T3DB, EPA ToxCast, and EPA DSSTox databases. Identifications were accepted for compounds based on their exact mass, isotope ratio, fragmentation, and retention time (if available). For the suspect screening approach, a special database was created to encompass compounds anticipated to be found in crumb rubber, based on analysis of the bulk material ${ }^{5}$ and other published information. Compounds were then search against this compiled database and identifications were accepted using the same criteria listed above.

Data were exported from Progenesis QI (Nonlinear Dynamics, UK) and imported into SIMCA 14.1 (Umetrics, Umeå, Sweden) to observe and identify any differentiating metabolites between treated and control groups. Unsupervised principal component analysis (PCA) was conducted on the datasets.

Data were exported from Progenesis QI (Nonlinear Dynamics, UK) for SAS analysis, and pvalues calculated using an Exact Wilcoxon Rank Sum Test. Fold changes were calculated for each compound by comparing the median values of the treated and untreated group. In addition, 
Synthetic Turf/Recycled Tire Crumb Rubber:

Characterization of the Biological Activity of Crumb Rubber In Vitro

where fold changes were not available for a compound (due to the presence of a near-zero median abundance in either the treated or untreated group), the median values of the non-zero group served as a "pseudo" fold change to capture changes. 


\section{Results}

Data include the salient findings from biological sample analysis and statistical or biologically relevant toxicological findings. Summary data and individual animal data for each endpoint can be found in the NTP Chemical Effects in Biological Systems database:

https://doi.org/10.22427/NTP-DATA-RR-14.

\section{Survival, Body Weights, and Clinical Observations}

Crumb rubber exposure had no effect on survival, body weight, or clinical observations following oral gavage, dosed feed, or mixed bedding exposure. Body weights were within $2 \%$ of the respective control group throughout the study for each route of exposure (Figure 3, Figure 4, and Figure 5). Transient observations of soiled or ruffled coat were noted in some gavage animals (treated and control), and was likely related to the corn oil vehicle used in those animals, and was not considered to be related to crumb rubber exposure.

Qualitatively, the feces from animals exposed to crumb rubber in feed and by oral gavage appeared darker in color (dark brown to black) compared to feces from control animals. In oral gavage animals, a few occurrences of dark feces were observed at several time points throughout the study. Feces from animals in the 50,000-ppm group appeared black on study Day 4. On subsequent observations, feces from these animals remained darker than those for bedding or gavage animals, but much lighter than Day 4 feces, suggesting an active avoidance of the crumb rubber within feed compared to early in the study. Feces color from animals in the mixed bedding study was unchanged.

\section{Food and Material Consumption}

Some statistically significant differences in food consumption were observed for each route of exposure, but none of these changes was considered biologically relevant. The differences observed were within an expected range of variability for this endpoint.

Quantifying the consumption of crumb rubber was attempted in the 50,000-ppm feed group. Traditionally in feed studies, the chemical of interest is adsorbed to feed particles and homogeneously distributed, such that chemical consumption can be calculated by changes in feed weight, before and after administration of dosed feed. Due to the larger size of crumb rubber particles compared to milled feed, animals could have actively avoided consuming the crumb rubber particles. Further, the crumb rubber did not adsorb to the feed, thereby making traditional methods to measure compound consumption impractical. To address the consumption issue, an attempt was made to separate the crumb rubber from feed post-administration and weigh the fractions separately. Prior to preparing feed formulations, aliquots of feed and crumb rubber were weighed separately and then when feeders were changed (Days 4, 7, 11), the contents were sieved to separate the feed and crumb rubber and re-weighed. Table 2 shows the weigh-in and weigh-back weights of feed and crumb rubber for the three cages in the 50,000-ppm group for each of the 3-day intervals that correspond with feeder changes. Because of fecal and bedding contamination of feeders, there were consistently inaccurate weigh-back weights for crumb rubber due to the size similarity of the feces, bedding, and crumb rubber. Based on this complication, quantitation of crumb rubber consumption was deemed infeasible. Qualitatively, 
based on fecal observations described above, animals in the 50,000-ppm feed group appeared to consume some crumb rubber; however, the change in fecal color from Days 0-4 and later in the study suggests an active avoidance of the crumb rubber particles.

\section{Assessment of Internal Exposure}

Based on an unsupervised PCA, of the plasma and urine data for each study matrix tested, separation between control and treated groups was not apparent for any route of exposure or matrix (Figure 6, Figure 7, and Figure 8).

The data for all matrices, exposure routes, and ionization modes (positive ion or negative ion), were subsequently analyzed (post processing in Progenesis) in SAS to calculate fold changes and p-values for well-detected features determined by Progenesis.

To initially filter data, a p-value $<0.1$ and fold change $>2$ (or $<-2$ ) were used to investigate differences between treated and control groups (https://doi.org/10.22427/NTP-DATA-RR-14). Following an initial screen, the data were filtered further using more strict criteria $(\mathrm{p}<0.05$, fold change >5) and features with a negative fold change (higher in controls than in treated) were not included. The focus of these studies was to identify potential constituents from the crumb rubber material in plasma and urine, rather than interpreting disruption of endogenous processes. For both the untargeted and suspect screening searches described below, database matching used exact mass and isotope ratio for $\mathrm{M}=1, \mathrm{M}+2$, etc., fragmentation (which for many databases are predicted fragments), and retention time. For each identified feature, other structures are possible; therefore $100 \%$ confidence in identity was not possible as standard reference samples were not used to confirm identification. In the hierarchy of identification for untargeted methods and unknowns, different confidence levels can be assigned depending on the extent of the $\operatorname{match}^{16}$.

Using an untargeted search against several databases listed above, a summary of the number of features with a p-value $<0.05$ and a fold change of $>5$ are shown in Table 3 for all matrices, exposure routes, and analysis modes. The crumb rubber gavage group had the highest number of differentially identified features compared to other routes, and more features were identified in plasma compared to urine for all routes of exposure. One feature was identified in plasma for all three routes of exposure with a p-value $<0.05$ and a fold change $>600$; this feature was tentatively identified as 6-acetamido-2-naphthalenesulfonic acid but was not confirmed with an authentic standard. No common features were identified in urine across exposure routes.

For the suspect screening approach, a database was constructed based on predicted mass, isotope ratios and authentic standards for compounds known to be found in crumb rubber. Very few of the features identified had fold changes $>5$, and only one had $\mathrm{p}<0.05$. In the gavage group, 1,2Dihydro-2,2,4-trimethylquinoline (CAS 147-47-4) was detected higher (12.6 fold, $\mathrm{p}=0.008$ ) in crumb rubber-exposed animals compared to controls. Several other compounds were detected with $\mathrm{p}=0.057$ with high fold changes in crumb rubber gavage animals compared to controls, including 2(3H)-benzothiazolethione (CAS 149-30-4; fold change 4,631.2) and neodecanoic acid (CAS 26896-20-8; fold change 3,259.4). These three compounds were all detected in the bulk crumb rubber material ${ }^{10}$. Using the suspect screening approach, there were no compounds identified in the feed or bedding groups that had $\mathrm{p}<0.05$ and fold change $>5$. 


\section{Organ Weights}

Organ weights for animals in the oral gavage study did not significantly change. Animals in the 50,000-ppm dosed feed group had significantly lower mean thymus $(-10 \%)$ and ovary $(-32 \%)$ weights compared to controls. Animals in the 50:50 wt:wt mixed bedding group had slightly higher, statistically significant, relative liver weight (6\%) compared to controls. These weight changes were not associated with any microscopic finding.

\section{Hematology and Bone Marrow Cytology}

Hematology values in the crumb rubber oral gavage study did not change relative to the control values. The neutrophil count was significantly lower in the 50,000-ppm dosed feed group. In the 50:50 wt:wt mixed bedding group, hematocrit was significantly lower, and the white blood cell and lymphocyte count significantly higher. These changes, however, were mild and not considered treatment related.

The M:E ratio of the bone marrow was unchanged in the dosed feed and oral gavage studies. The $\mathrm{M}: \mathrm{E}$ ratio of the 50:50 wt:wt mixed bedding group was significantly higher but was not considered a treatment-related change, in part because the control group's M:E ratio was substantially lower than would be normally expected (0.8-2.8 with an average of 1.5$)^{17}$.

\section{Histopathology}

There were no gross observations at necropsy and no histopathologic lesions were observed in animals exposed to crumb rubber via dosed feed (50,000 ppm) or mixed bedding (50:50 wt:wt).

Animals dosed with crumb rubber by oral gavage $(1,250 \mathrm{mg} / \mathrm{kg} /$ day) for 14 days had occurrences of esophageal inflammation in the control (4/10) and dosed (6/10) groups, however the incidence and average severity (minimal) of this lesion was similar between control and crumb rubberexposed groups. This lesion was not observed in other groups that were not gavage dosed. 
Synthetic Turf/Recycled Tire Crumb Rubber:

Characterization of the Biological Activity of Crumb Rubber In Vitro

\section{Discussion}

The objective of the studies described herein was to ascertain evidence of systemic exposure to crumb rubber constituents in vivo following dosing with crumb rubber. These studies aimed to provide data in a timely manner, and in coordination with other ongoing research efforts of crumb rubber under the FRAP and by OEHHA. These studies were not designed to generate dose-response information, address specific adverse outcomes (e.g., cancer or reproductive effects) or to investigate potential health for sensitive populations (e.g., pregnant women or children).

In these studies, female mice were exposed to crumb rubber via mixed bedding or administration in dosed feed or by oral gavage. These routes of exposure were selected based on feasibility testing that evaluated challenges associated with study design and conduct ${ }^{12}$. To some extent, the 14-day in vivo studies were an extension of the feasibility testing, to address the practicality of exposing animals to this unique material by various exposure routes. These studies help inform how subsequent in vivo studies should be conducted. While administration of crumb rubber was well tolerated in female mice by all routes tested, not all would be deemed successful or worthwhile to consider for future studies. Due to apparent avoidance of crumb rubber in the dosed feed study, accurately assessing systemic exposure to crumb rubber constituents was not feasible and would limit interpretation of dose response and toxicity if used for future studies. Bedding studies offer several advantages in terms of ease of study conduct, but have limitations with regards to known dose and low exposure. The most feasible and practical route of exposure for testing this material for future toxicity testing would be gavage dosing; this was the only route tested that allowed a quantifiable dose of crumb rubber particles.

To address the objective of these studies, evidence of systemic exposure to crumb rubber constituents was evaluated using untargeted mass spectrometry analysis of urine and plasma, and by using conventional toxicological endpoints to serve as evidence of biological effect. Treatment-related effects were not observed for survival, body weight, organ weights, clinical observations, hematology, bone marrow cytology or histopathology following any route of exposure. Evaluation of urine and plasma profiles revealed no separation between treatment and control groups, for any route of exposure. This could indicate there was no appreciable exposure to crumb rubber constituents or that high variability between animals within a given group obscured any effect between exposed and control groups. A higher number of differentially expressed features between exposed and control groups were observed in animals from the gavage study compared to other routes. This is consistent with the known exposure/dosing of these animals, compared to lower exposure in the bedding, and unknown exposure in feed animals. Using database matches against known crumb rubber constituents, one compound was tentatively identified (1,2-Dihydro-2,2,4-trimethylquinoline; DHTMQ) that met our criteria ( $p<0.05$, fold change $>5$ ). This compound was also identified (by library match) in the NTP bulk crumb rubber material using methylene chloride extraction and gas chromatography mass spectrometry analysis ${ }^{10}$. DHTMQ is used as an antioxidant in styrene-butadiene and nitrilebutadiene rubbers and latexes and has previously been studied by NTP in 2-year dermal studies in rats and mice ${ }^{18}$. Studies in female rats and male and female mice were negative. In male rats there were incidences of renal tubule adenomas and carcinomas that were considered to be related to treatment. 
Overall, based on analysis of urine and plasma, and traditional toxicological endpoints, there was little signal that would indicate systemic exposure to crumb rubber constituents under the various conditions employed in these studies. In parallel to conducting these studies, a robust chemical analysis of the bulk material was completed that provides additional context for the findings from the 14-day in vivo studies. In our chemical analysis of the bulk material ${ }^{10}$, the volatile and semivolatile compounds, and metals are present at low levels. For example, the highest concentration volatile constituents were present at $3.35,1.05$, and $0.72 \mathrm{ppm}$ (methyl isobutyl ketone, aniline and benzothiazole, respectively). This means that even if efficient extraction of crumb rubber constituents occurred within an animal (e.g., $100 \%$ bioavailability), the amount of a given compound available for systemic circulation would be very low. Based on our understanding of leachability using simulated biological fluids ${ }^{10}$, extensive extraction of constituents is unlikely under physiological conditions. Given these assumptions, it is not surprising there was limited evidence of systemic exposure to crumb rubber constituents in the in vivo studies. These assumptions also imply that our ability to detect many of these constituents by chemical analysis of biological samples from exposed animals would also be limited. However, based on the toxicological potency of some constituents, or interaction of multiple constituents, it is likely that the toxicological endpoints evaluated in these studies would have shown a treatment-related effect if biological perturbation occurred following systemic exposure. 


\section{Conclusions}

There was little evidence of systemic exposure to crumb rubber constituents in female mice following administration by oral gavage, in dosed feed or mixed bedding. While there was little evidence of exposure, the analysis of urine and plasma will help inform human biomonitoring activities by providing potential targets that can be pursued with targeted/validated methods. The data presented herein and in supplementary files (https://doi.org/10.22427/NTP-DATA-RR-14) also represent the only hazard identification-type study of this unique material and provide a valuable resource for researchers in the design and conduct of future toxicology studies of crumb rubber. 


\section{References}

1. Perkins AN, Inayat-Hussain SH, Deziel NC, Johnson CH, Ferguson SS, Garcia-Milian R, Thompson DC, Vasiliou V. Evaluation of potential carcinogenicity of organic chemicals in synthetic turf crumb rubber. Environ Res. 2019; 169:163-172.

https://doi.org/10.1016/j.envres.2018.10.018

2. United States Environmental Protection Agency (U.S. EPA). Federal research action plan (FRAP) on recycled tire crumb used on playing fields and playgrounds. 2016. EPA/600/R16/364. https://www.epa.gov/sites/production/files/201612/documents/federal_research_action_plan_on_recycled_tire_crumb_used_on_playing_fields_a nd_playgrounds_status_report.pdf [Accessed: 3 Oct, 2017]

3. European Chemicals Agency (ECHA). Annex XV Report: An evaluation of the possible health risks of recycled rubber granules used as infill in synthetic turf sports fields. 2017. https://echa.europa.eu/documents/10162/13563/annexXV_report_rubber_granules_en.pdf/dbcb4ee6-1c65-af35-7a18-f6ac1ac29fe4

4. Rijksinstituut voor Volksgezondheid en Milieu [Netherlands National Institute for Public Health and the Environment] (RIVM). Evaluation of health risks of playing sports on synthetic turf pitches with rubber granulate. 2017. Report Number: 2017-0016. https://www.rivm.nl/dsresource?objectid=a397bdf3-7aa7-490a-85bd5992b78bcb42\&type $=$ pdf \&disposition $=$ inline

5. United States Environmental Protection Agency (U.S. EPA). Federal research on recycled tire crumb used on playing fields. U.S. Environmental Protection Agency; 2016.

https://www.epa.gov/chemical-research/federal-research-recycled-tire-crumb-used-playing-fields [Accessed: 2017]

6. Agency for Toxic Substances and Disease Registry (ATSDR). Federal research action plan on recycled tire crumb used on playing fields and playgrounds. 2016.

https://www.atsdr.cdc.gov/frap/index.html [Accessed: 2017]

7. Consumer Product Safety Commission (CPSC). Crumb rubber information center. 2016. https://www.cpsc.gov/Safety-Education/Safety-Education-Centers/Crumb-Rubber-SafetyInformation-Center [Accessed: 2017]

8. Office of Environmental Health Hazard Assessment (OEHHA). Synthetic turf studies. 2017. https://oehha.ca.gov/risk-assessment/synthetic-turf-studies [Accessed: 2017]

9. National Toxicology Program (NTP). Synthetic turf/recycled tire crumb rubber. 2017. https://ntp.niehs.nih.gov/results/areas/syntheticturf/index.html [Accessed: 2017]

10. National Toxicology Program (NTP). NTP research report on chemical and physical characterization of crumb rubber. Research Triangle Park, NC: National Toxicology Program; 2019. Research Report 11. https://doi.org/10.22427/NTP-RR-11

11. National Toxicology Program (NTP). NTP research report on synthetic turf/recycled tire crumb rubber: Characterization of the biological activity of crumb rubber in vitro. Research 
Triangle Park, NC: National Toxicology Program; 2019. Reserch Report 12. https://doi.org/10.22427/NTP-RR-12

12. National Toxicology Program (NTP). NTP research report on synthetic turf/recycled tire crumb rubber: Feasibility study in support of non-inhalation in vivo exposures of synthetic turf/recycled tire crumb rubber. Research Triangle Park, NC: National Toxicology Program; 2019. Research Report 13. https://doi.org/10.22427/NTP-RR-13

13. National Toxicology Program (NTP). NTP research report on synthetic turf/recycled tire crumb rubber: 14-day exposure characterization studies of crumb rubber in female mice housed on mixed bedding or dosed via feed or oral gavage. Research Triangle Park, NC: National Toxicology Program; 2019. Research Report 14. https://doi.org/10.22427/NTP-RR-14

14. National Research Council (NRC). Guide for the care and use of laboratory animals. 8th ed. Washington, DC: NRC (US) Committee for the Update of the Guide for the Care and Use of Laboratory Animals; 2011.

15. National Toxicology Program (NTP). (National Toxicology Program). Specifications for the conduct of studies to evaluate the toxic and carcinogenic potential of chemical, biological and physical agents in laboratory animals for the National Toxicology Program (NTP). 2011. https://ntp.niehs.nih.gov/ntp/test_info/finalntp_toxcarspecsjan2011.pdf

16. Schymanski EL, Singer HP, Slobodnik J, Ipolyi IM, Oswald P, Krauss M, Schulze T, Haglund P, Letzel T, Grosse S et al. Non-target screening with high-resolution mass spectrometry: Critical review using a collaborative trial on water analysis. Anal Bioanal Chem. 2015; 407(21):6237-6255. https://doi.org/10.1007/s00216-015-8681-7

17. Everds NE. Hematology of the laboratory mouse In: Fox JG, editor. The Mouse in Biomedical Research. Waltham, MA: Academic Press; 2007. p. 133-170.

https://doi.org/10.1016/B978-012369454-6/50059-5

18. National Toxicology Program (NTP). NTP toxicology and carcinogenesis studies of 1,2dihydro-2,2,4-trimethylquinoline (CAS No. 147-47-7) in F344/N rats and B6C3F1 mice (dermal studies) and the dermal initiation/promotion study in female Sencar mice. Research Triangle Park, NC; 1997. Technical Report 456. 
Synthetic Turf/Recycled Tire Crumb Rubber:

Characterization of the Biological Activity of Crumb Rubber In Vitro

Table 1. Crumb Rubber Particle Size Summary ${ }^{a}$

\begin{tabular}{lcccc}
\hline \multicolumn{1}{c}{ Study } & $\begin{array}{c}\text { Size Range } \\
(\boldsymbol{\mu \mathbf { m } )}\end{array}$ & Average Length $(\boldsymbol{\mu m})^{\mathbf{a}}$ & $\begin{array}{c}\text { Length Range } \\
(\boldsymbol{\mu \mathbf { m }})^{\mathbf{a}}\end{array}$ & \% Total by Weight \\
\hline Oral Gavage & $37-170^{\mathrm{b}}$ & 46.0 & $8.3-167$ & 0.34 \\
Dosed Feed & Greater than $170^{\mathrm{c}}$ & 2,135 & $500-4,400$ & 99.66 \\
Mixed Bedding & & & & \\
\hline
\end{tabular}

${ }^{a}$ Size characterization was performed on 65 and 23 particles for the less than $37-170 \mu \mathrm{m}$ and greater than $170 \mu \mathrm{m}$ size fractions, respectively.

${ }^{b}$ Lot no. CRM09132016; mesh size 400.

'Lot no. CRM12052016; mesh sizes 14-80. 
Synthetic Turf/Recycled Tire Crumb Rubber:

Characterization of the Biological Activity of Crumb Rubber In Vitro

Table 2. Consumption of Crumb Rubber in 50,000 ppm Dosed Feed Group

\begin{tabular}{ccccc}
\hline Cage & \multicolumn{2}{c}{ Feed (g) } & \multicolumn{2}{c}{ Crumb Rubber (g) } \\
\hline Day 0 to 4 & Weigh In & Weigh Back [\% change] & Weigh In & Weigh Back ${ }^{\mathrm{a}} \%$ change] \\
$\mathbf{9}$ & 120.00 & $45.18[-62]$ & 5.99 & $16.50[+175]$ \\
$\mathbf{1 0}$ & 120.24 & $43.27[-64]$ & 6.00 & $22.66[+277]$ \\
$\mathbf{1 2}$ & 120.70 & $39.81[-67]$ & 6.01 & $28.92[+381]$ \\
Day 4 to 7 & & & & \\
$\mathbf{9}$ & 120.75 & $56.48[-53]$ & 6.04 & $26.45[+221.8]$ \\
$\mathbf{1 0}$ & 119.80 & $56.83[-53]$ & 6.03 & $30.12[+400]$ \\
$\mathbf{1 2}$ & 120.50 & $66.25[-45]$ & 6.02 & \\
Day 7 to 11 & & & 6.01 & $25.55[+329]$ \\
$\mathbf{9}$ & 119.90 & $46.53[-61.2]$ & 6.02 & $25.38[+322]$ \\
$\mathbf{1 0}$ & 120.44 & $45.09[-63]$ & 6.03 & $26.05[+332]$ \\
$\mathbf{1 2}$ & 119.79 & $41.11[-65.7]$ & & \\
Day 11 to 14 & & & 6.00 & $26.30[+338]$ \\
$\mathbf{9}$ & 119.85 & $57.87[-52]$ & 6.03 & $29.73[+393]$ \\
$\mathbf{1 0}$ & 120.43 & $53.60[-56]$ & 6.00 & $25.32[+322]$ \\
$\mathbf{1 2}$ & 119.77 & $66.59[-44]$ & & \\
\hline
\end{tabular}

${ }^{\mathrm{a}}$ Crumb rubber and other constituents of similar size (bedding and feces).

bValues are for Days 11-13 due to removal of biological sampling mice on Day 13. 
Synthetic Turf/Recycled Tire Crumb Rubber:

Characterization of the Biological Activity of Crumb Rubber In Vitro

Table 3. Untargeted Analysis ${ }^{\mathrm{a}}$

\begin{tabular}{lcccccc}
\hline Ion Mode & \multicolumn{2}{c}{ Oral Gavage } & \multicolumn{2}{c}{ Dosed Feed } & \multicolumn{2}{c}{ Mixed Bedding } \\
\hline & Plasma & Urine & Plasma & Urine & Plasma & Urine \\
Positive & 25 & 0 & 5 & 0 & 2 & 1 \\
Negative & 13 & 1 & 2 & 0 & 4 & 1 \\
\hline
\end{tabular}




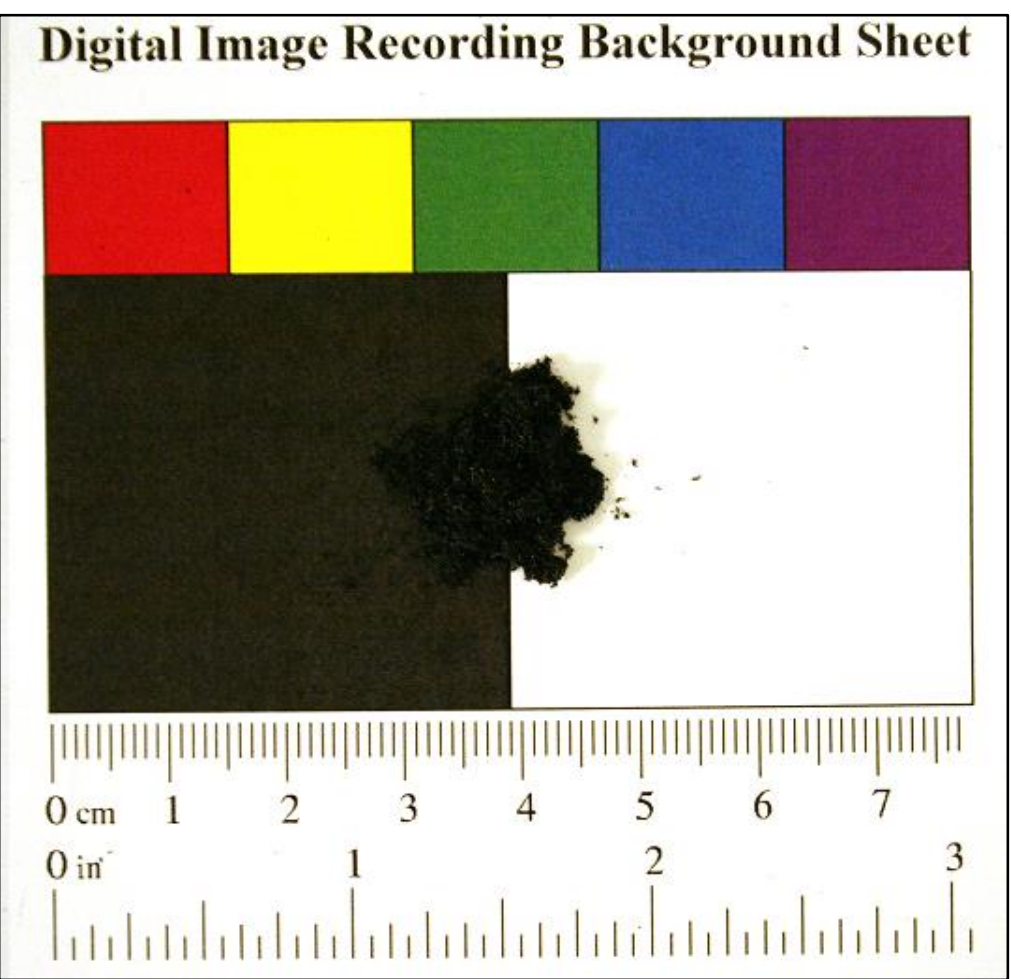

Figure 1. Image of Crumb Rubber Particles: Lot CRM09132016, 400 Mesh

\section{Digital Image Recording Background Sheet}

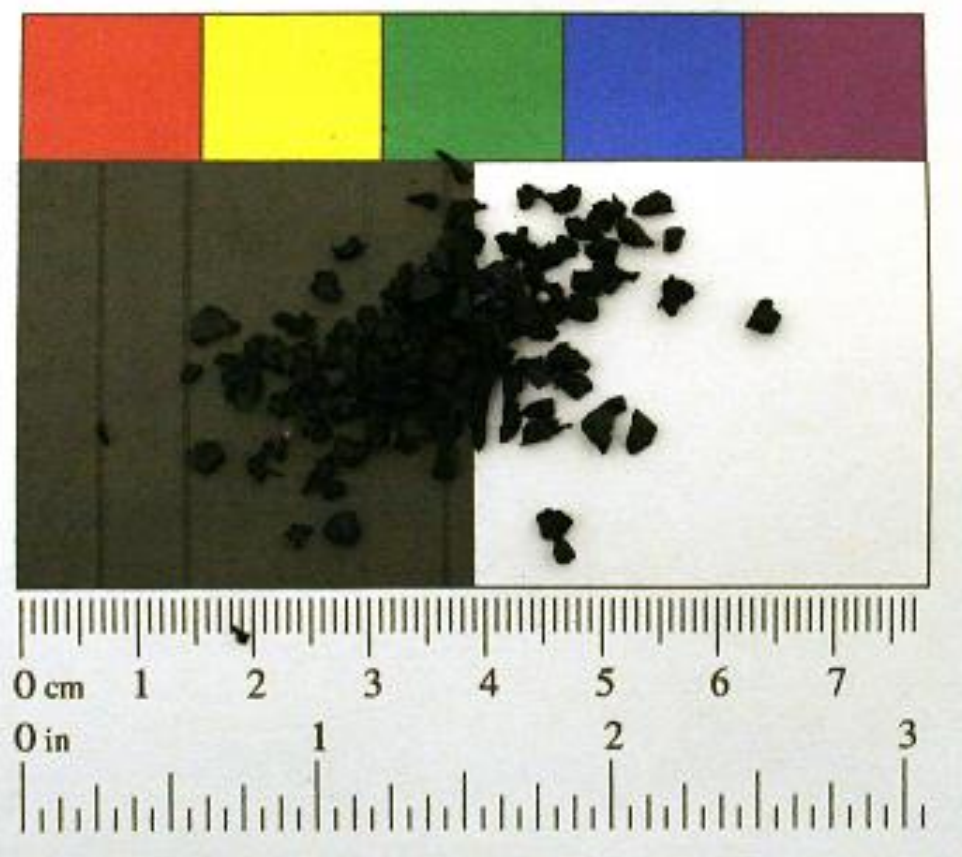

Figure 2. Image of Crumb Rubber Particles: Lot CRM 12052016, Combined 


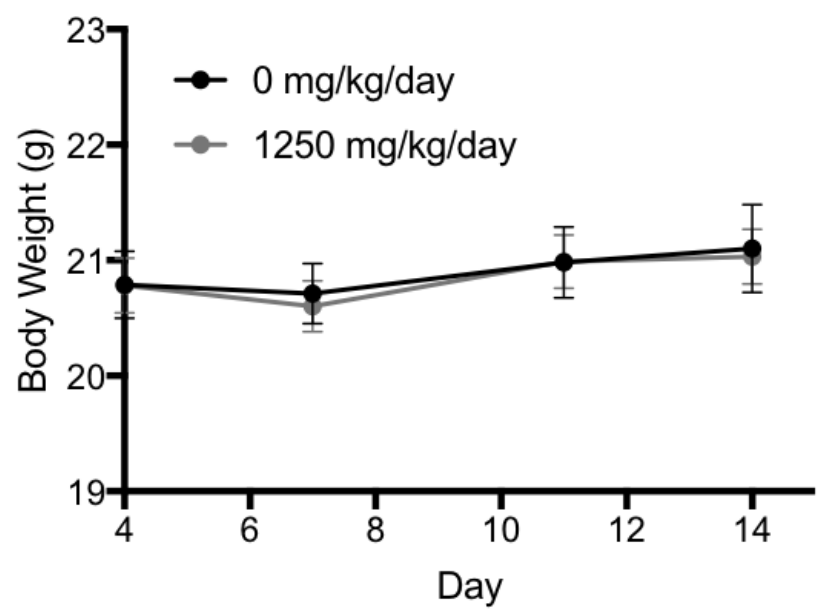

Figure 3. Body Weight; Oral Gavage Study

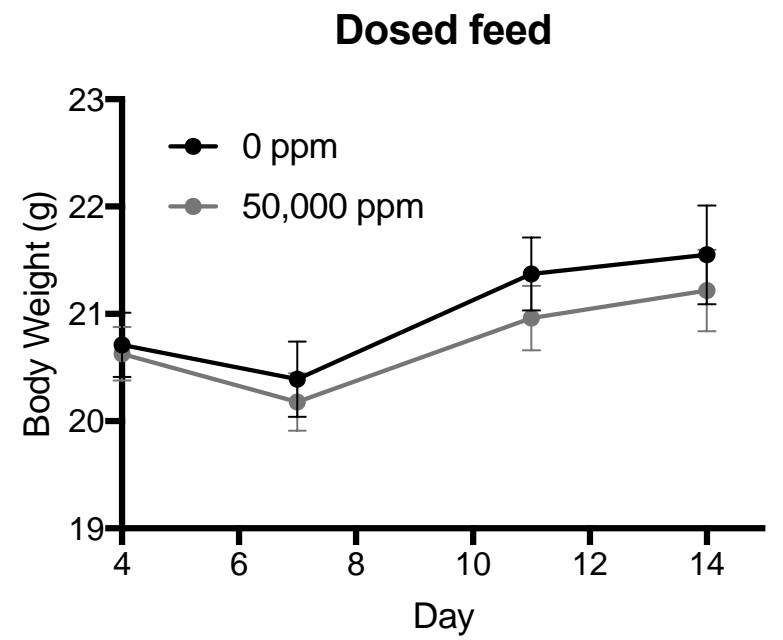

Figure 4. Body Weight; Dosed Feed Study

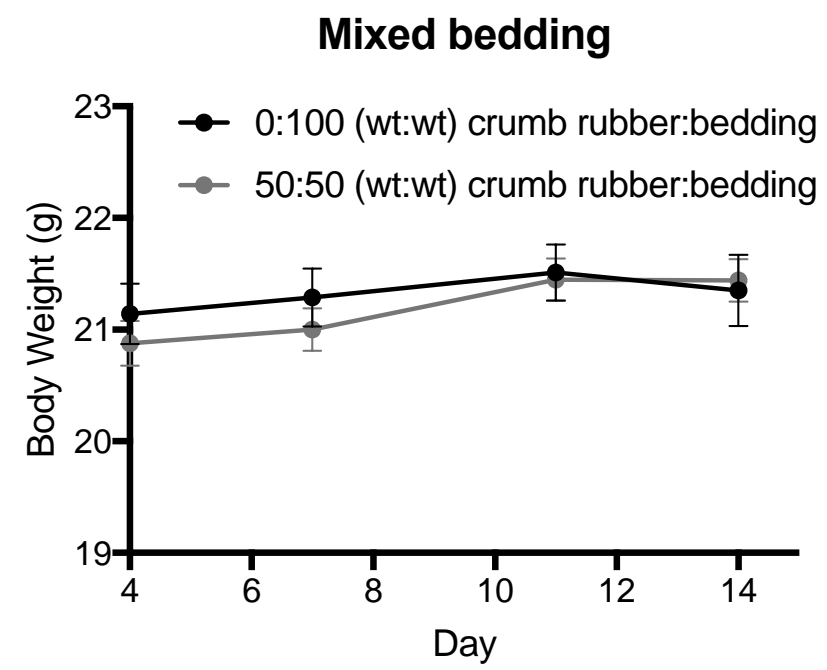

Figure 5. Body Weight; Mixed Bedding Study 
Synthetic Turf/Recycled Tire Crumb Rubber:

Characterization of the Biological Activity of Crumb Rubber In Vitro
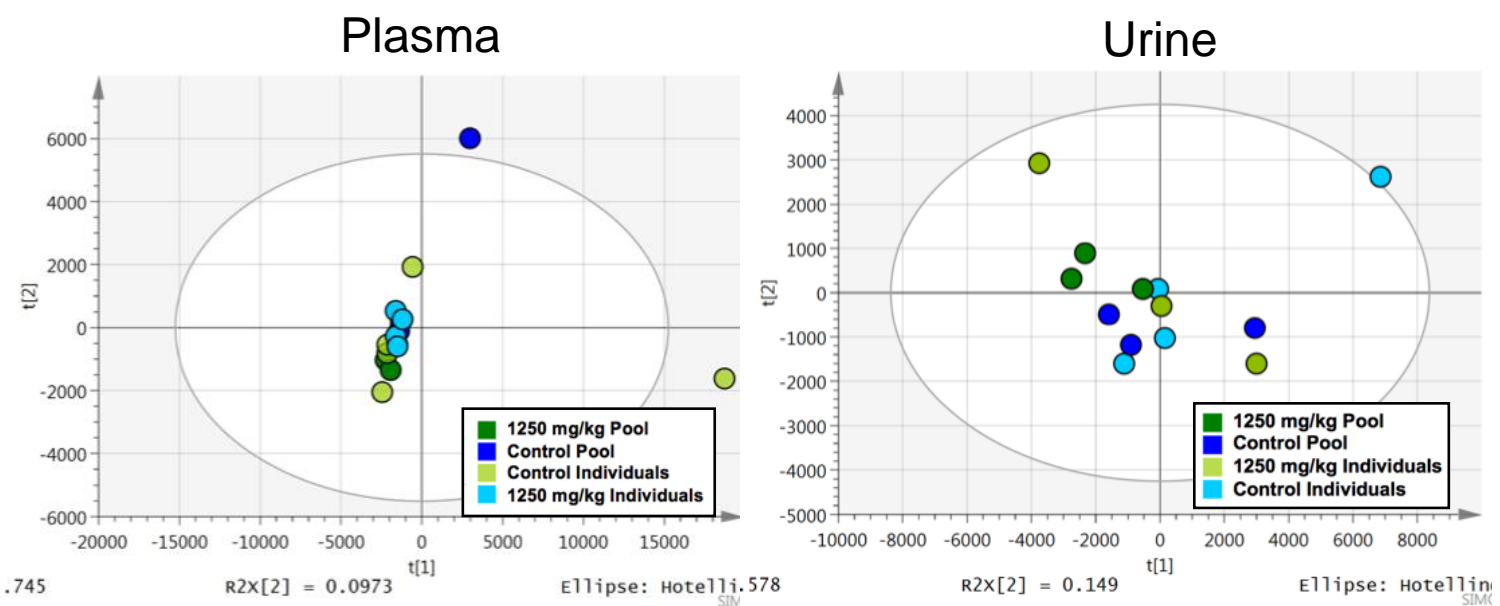

Figure 6. Principal Component Analysis of Plasma and Urine Untargeted Data; Oral Gavage Study
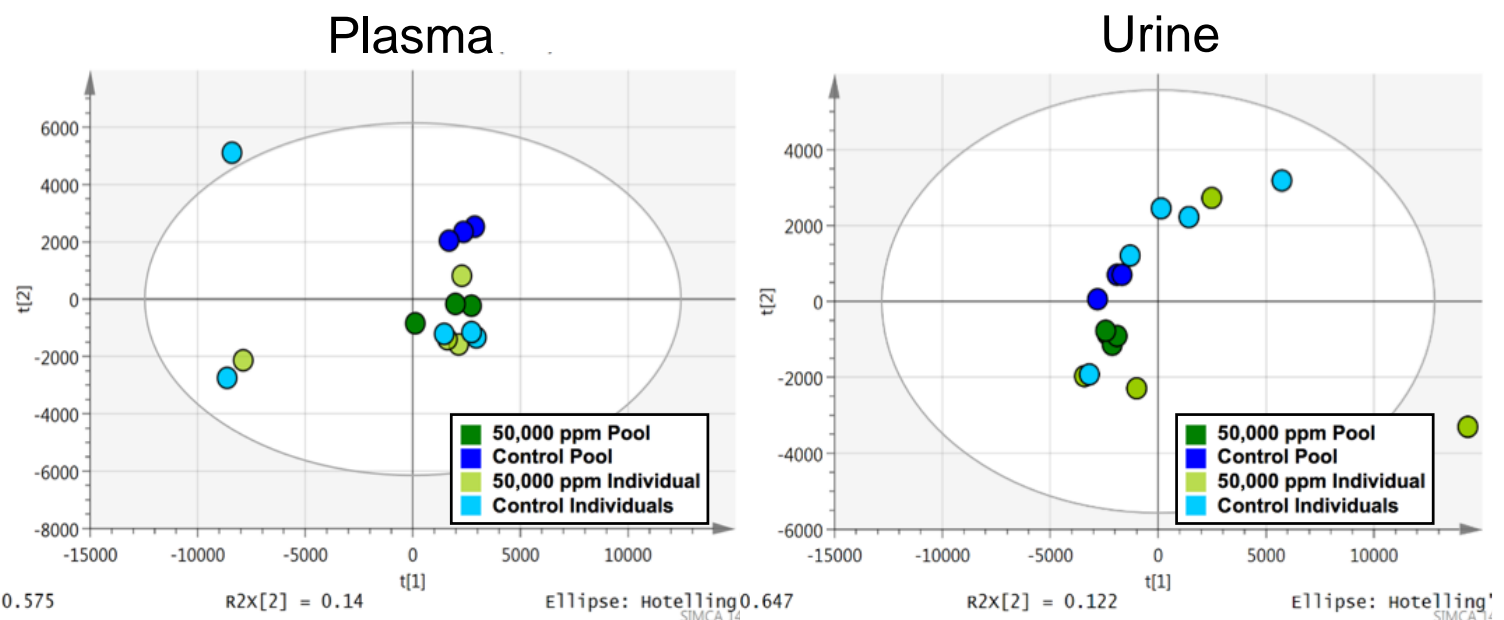

Figure 7. Principal Component Analysis of Plasma and Urine Untargeted Data; Dosed Feed Study
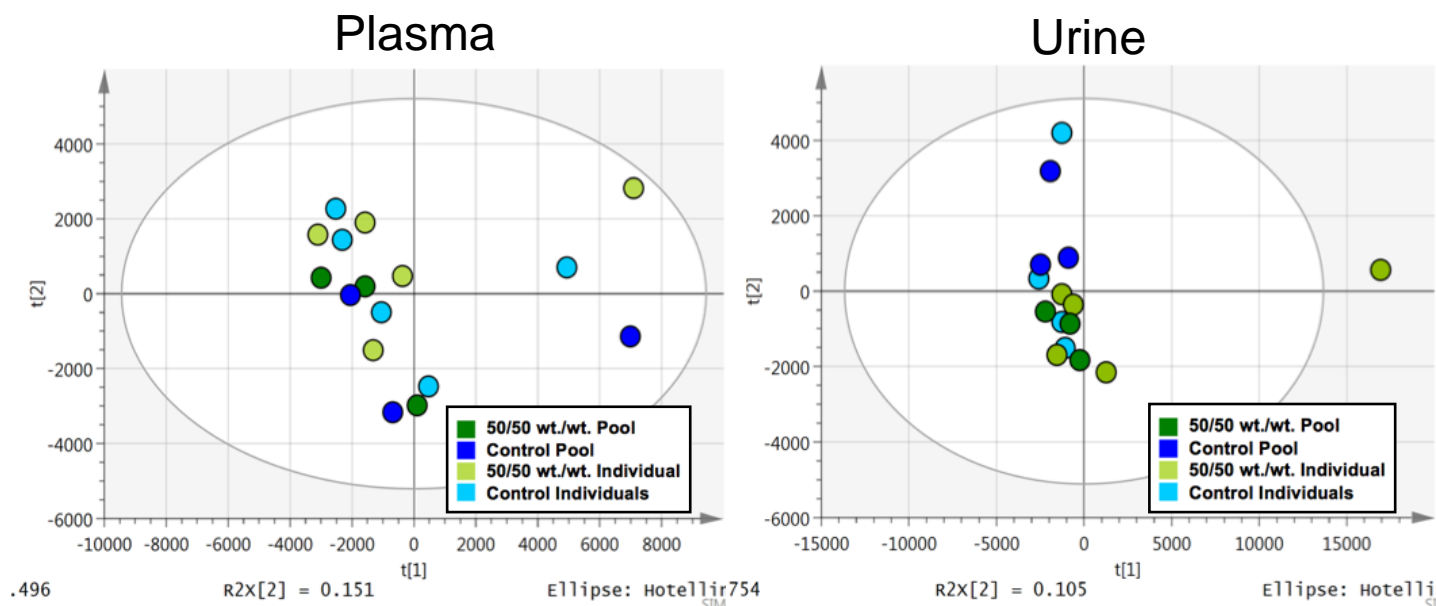

Figure 8. Principal Component Analysis of Plasma and Urine Untargeted Data; Mixed Bedding Study 


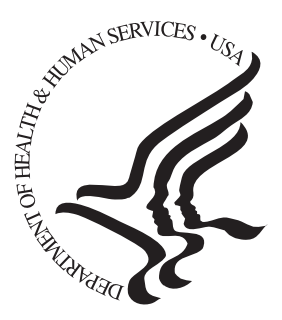

\section{National Toxicology Program}

NTP Central Data Managment, MD K2-05

National Insitute of Enviromental Health Sciences

P.O. Box 12233

Research Triangle Park, NC 27709

http://ntp.niehs.nih.gov 